\title{
Review of 48 cases of motor neuron disease seen at groote schuur hospital: a pilot study
}

\begin{abstract}
Background: Motor neuron disease (MND) is a rare neurodegenerative disorder that causes progressive weakness of the limb, bulbar and respiratory muscles and results in the death of the patient. The demography of MND in South Africa, in particular, was not studied previously. The study aimed to describe the demographics and clinical characteristics of MND seen at Groote Schuur Hospital (GSH).

Methods: It was a retrospective review of the patients with MND presented to GSH from January 2005 to December 2010. El Escorial diagnostic criteria were used to check the validity of the diagnosis of MND. Mortality data were obtained from the Medical Research Council of South Africa.

Results: Forty-eight patients met the El Escorial criteria. The median age of onset of the disease was 54-year (IQR 47-63), and the median duration of the disease from the earliest symptoms to death was 2-year (IQR 1-3). There was no significant difference between the bulbar and limb-onset disease sub-types. There was a male preponderance $(60 \%)$, and the majority of the patients $(60 \%)$ were smokers. Exposure to chemicals was disproportionately represented in the MND patients compared to the general population. Baseline CSF-protein and serum Creatinine Phosphokinase levels were not associated with disease duration.

Conclusion: The characteristics of MND cases seen at GSH are similar to those in the Europe or North America. This pilot study will serve as the basis for future larger prospective studies on MND prevalence and etiology in South Africa.
\end{abstract}

Volume 7 Issue 6 - 2017

\author{
Amina Daude,' Sharfuddin Chowdhury² \\ 'Department of Medicine, Klerksdorp/Tshepong Hospital \\ Complex, South Africa \\ ${ }^{2}$ Consultant and Head of Trauma, King Saud Medical City, \\ Kingdom of Saudi Arabia
}

\author{
Correspondence: Sharfuddin Chowdhury, Trauma Unit, \\ General Hospital, King Saud Medical City, 779 Al-Imam Abdul \\ Aziz Ibn Muhammad Ibn Saud, Ulaishah, Riyadh I2746, Kingdom \\ of Saudi Arabia,Tel +966 || 4355555 Ext: | 385, \\ Emaildr_smahmud@yahoo.com
}

Received:September 27, 2017 | Published: October 30, 2017

\section{Introduction}

Motor neuron disease (MND) is a group of neurodegenerative disorders related to selective degeneration of motor neurons in the brain and spinal cord. Studies of the incidence, prevalence and demographic characteristics of patients with MND have mostly been done in the industrialized countries. We know petite about the disease in Africa in general and southern Africa in particular. Our anecdotal evidence from the neurology and geriatric medicine clinics suggests that the disease is not uncommonly seen in younger patients.

The study aimed to describe the demographics and clinical characteristics of MND seen at Groote Schuur Hospital (GSH). The objective was to compare some features of MND seen locally with the traits derived from the studies in the developed world. We were also designed to test some hypotheses based on our clinical observations of the cases of MND seen in the Western Cape.

\section{Methods}

This pilot study was a Human Research Ethics Committee (HREC) approved retrospective review of MND patients, identified from the database in the Division of Neurology, Department of Medicine, GSH and University of Cape Town (UCT) from 2005 to 2010.

Data were collected from the clinical records to characterize the cases of MND on the age of onset of the disease, duration of symptoms, sex, population group, MND type/form of the disease, possible exposures to toxins, place of residence, and death. The laboratory values including plasma creatine phosphokinase (CPK) levels, erythrocyte sedimentation rate (ESR), white cell count (WCC), and cerebrospinal fluid (CSF) protein concentration were also studied. To find the records of the death involved the searching of the database of the registration of births and deaths of the Department of Home
Affairs of South Africa. It was done through the South African Medical Research Council (MRC) Burden of Diseases Unit.

The cases were classified according to the El Escorial diagnostic criteria, EEDC (definite, possible and probable) at first presentation.

\section{Hypotheses $(\mathrm{H})$ tested}

H1. The age of onset of MND is earlier in the Western Cape population compared to other population studies. Also, black African patients have a younger age of onset of MND than populations of European ancestry.

$\mathrm{H} 2$. There is a male preponderance of the disease.

H3. A disproportionate number of cases are seen from the West Coast region compared to other areas of the Western Cape.

H4. Younger onset disease has a shorter duration of symptoms to death.

H5. The age of onset is earlier, and the length of illness is more succinct in familial MND than sporadic MND.

H6. Bulbar onset disease has a shorter survival compared to the limb-onset disease.

H7. Occupations that involve exposure to agricultural and other chemicals will be disproportionately represented in the MND patients.

H8. CPK levels will be higher in MND patients than normal control laboratory values.

H9. High CPK levels will be associated with faster disease progression and time to death.

H10. Both peripheral blood and CNS inflammatory markers will be raised in MND patients.

H11. Cigarette smoking is related to an increased risk of MND. 
Many of these hypotheses are based on the previously reported findings, or putative associations suggested in the literature. H1 and $\mathrm{H} 3$ are based on anecdotal observations by clinicians at GSH, but they require a more rigorous examination.

\section{Statistical analysis}

Most data were not normally distributed; therefore, descriptive data were presented as a median and interquartile range (IQR). Specific statistical tests including Kruskal-Wallis, Pearson Product Moment correlation, Spearman rank correlation and Mann-Whitney U test (where data were not normally distributed) were used for the different hypotheses testing. Significance was set at the 0.05 level.

\section{Results}

A total of 50 patients were recognized with the diagnosis of MND in the database. However, two were excluded from the analyses as one had missing notes, and the other, on review, did not meet the EEDC for the diagnosis of MND. The clinical records of 48 patients were examined. The descriptive data are summarized in Table 1.

Table I Demographic characteristics and MND subtypes of 48 patients included in the study

\begin{tabular}{ll}
\hline Characteristics & $\mathbf{n}(\%)$ \\
\hline Sex & \\
Male & $29(60 \%)$ \\
Female & $19(40 \%)$ \\
Population Classification & \\
Mixed ancestry & $29(60 \%)$ \\
Black African & $1 \mathrm{I}(23 \%)$ \\
White & $8(\mathrm{I} \% \%)$ \\
Median age of onset of symptoms & $54(\mathrm{IQR} 47.5-63)$ \\
Median age of death & $56(\mathrm{IQR} 47-65)$ \\
Median duration (in years) of disease (onset to death) & $2(\mathrm{IQR} \mathrm{I}-3)$ \\
Subtypes of MND & \\
Bulbar & $38(79 \%)$ \\
PMA & $7(\mathrm{I} \% \%)$ \\
PLS & $3(6 \%)$ \\
EEDC & \\
Clinically definite & $\mathrm{I}(3 \mathrm{I} \%)$ \\
Clinically probable & $24(50 \%)$ \\
Clinically possible & $9(\mathrm{I} \% \%)$ \\
\hline
\end{tabular}

MND: Motor Neuron Disease; PMA: Progressive Muscular Atrophy; PLS: Progressive Lateral Sclerosis; EEDC: EI Escorial Diagnostic Criteria

The median age of onset of MND was 54 years (IQR $47.5-$ 63.0) and the duration of the disease until death was two years (IQR 1-3) which did not show the statistically significant difference by population group compared to published literature $(p=0.057$, and $\mathrm{p}=0.39$ respectively). There was also no association between the age of onset and the disease duration $(\mathrm{p}=0.113)$. There was a tendency for a male preponderance $(\mathrm{M}: \mathrm{F}=3: 2)$ of the disease $(\mathrm{p}=0.056)$. A disproportionate number of cases were seen from the West Coast region $(n=3,6.25 \%)$ compared to the other areas of the Western Cape $(\mathrm{p}=0.46)$. The mean age of onset for the familial MND (fMND) was $55.8 \mathrm{yr}$. and for the sporadic MND (sMND) was $54.2 \mathrm{yr}$. The age of onset of the MND was not significantly different between the three subtypes- bulbar, progressive muscular atrophy (PMA), and progressive lateral sclerosis (PLS). There was a disproportionate number of MND patients $(n=9,18.75 \%)$ relative to the general population, in the Western Cape, who worked in the chemical and agricultural industries $(8.05 \%)(\mathrm{p}=0.0032)$. The MND is associated with higher plasma CPK levels (mean 230.6, SD 204.9) compared to the normal control population $(\mathrm{p}<0.0001)$. The Higher CPK levels were not associated with the faster disease progression and time to death $(0.05<\mathrm{p}<0.10)$. The MND patients tended to have higher than normal $(16.7 \%>20)$ ESR levels $(\mathrm{p}=0.0008)$. Forty $(83.33 \%)$ patients had the normal WCC and the rest $(2.1 \% \mathrm{WCC}<4$, and $14.6 \% \mathrm{WCC}$ $>11)$ had outside the normal range $(\mathrm{p}=0.0008)$. The CSF protein levels were frequently too high $(27.9 \%>0.45)$ or too low $(20.8 \%<0.2)$ $(\mathrm{p}<0.0001)$ (Table 2). The MND patients have significantly higher (men $75.9 \%$ and women $68.4 \%$ compared to men $44.7 \%$ and women $27 \%$ of normal population in the Western Cape ${ }^{1}$ ) smoking rates than expected, for both men $(p=0.0004)$ and women $(p<0.0001)$. The results of the hypotheses are summarised in Table 3.

\section{Discussion}

Anecdotally, we thought that we saw many younger-onset MND cases at GSH. However, our mean age of onset was similar to the report in the literature. A study in Nigeria in 2004 showed an average age of onset of 38.6 years. ${ }^{2}$ Many cases of spinal muscular atrophy may have been included in their study. The neurologists at GSH also thought that they were seeing more young black African patients with early onset MND. The mean age of onset in "African" patients (45.9 years) was lower than that in patients with European ancestry (58.8 years). With a larger number of participants, we might have demonstrated a significant difference. These results might then support the findings from Nigeria that MND onset in black Africans is, on average, lower than that described in studies from Europe and North America. A population-based mortality study from Cuba found that disease rates were $60 \%$ lower in their population compared to European and North American populations. ${ }^{3}$

The reason for the male preponderance is not known. Perhaps men are more exposed to the occupational hazards that are associated with MND, e.g., work in the chemical industries. There is a variant of PMA known as Kennedy's syndrome or bulbospinal muscular atrophy, which is linked to a trinucleotide expansion repeat in the testosterone receptor gene ${ }^{4}$. Hormonal influences may therefore also, be important in explaining the gender differences in MND.

Anecdotal evidence from neurologists has suggested that there might be clustering of cases in this particular area of the Western Cape Province. Our analysis showed that the proportion of patients from this area were consistent with the percentage of people from the Western Cape, who live in the West Coast area. That is, they were not disproportionately represented in the MND patients. However, another important factor is the drainage area of the GSH. There is another large academic hospital in the Western Cape with a tertiary neurology service: the Tygerberg Hospital (TBH). It also drains a larger number of patients from this area. Ideally, we should perform a larger study that includes cases in the same period seen at both GSH and TBH. In so doing, we should then be able to derive real prevalence data of MND in the Western Cape. Another factor is the place of residence of the patients that was gathered from the address in the hospital folder. However, people migrate, and there may be patients, who live in, say, the Mitchell's Plain District of the Western Cape now, who might have spent most of their lives on the West Coast. Again, in an ideal prospective study, we should ascertain not just their current address, but the places they lived in for the majority of their lives.

The fact that younger-onset disease was not associated with a shorter duration of symptoms to death has previously been reported by Li et al., ${ }^{5}$ Again, larger numbers might have shown this association, but our study did not support $\mathrm{H} 4$.

We had a similar proportion of fMND cases that have been reported worldwide. A study by Li et al., 5 showed an earlier age of onset, 
and a shorter duration of disease, in familial compared to sporadic MND. ${ }^{5}$ We did not perform any genetics studies on the familial cases to screen for known gene mutations associated with familial MND. Familial cases are rare, and the study is not sufficiently powered to show significant differences in age of onset and disease duration in familial $v s$. sporadic MND. Also, family histories may be incomplete, and many familial cases may not be recognized as such.

Table 2 Inflammatory CNS markers

\begin{tabular}{llll}
\hline Blood Markers & Distribution Skewed (s)/ normal(n) & $\mathbf{N = 4 8}$ Mean \pm SD & NHLS Lab Reference Range \\
\hline CPK & s & $230.6 \pm 204.9$ & Male: $26-140 \mathrm{IU} / \mathrm{ml}$ \\
WCC & s & $68 \pm 4.2$ & Female: $38-174 \mathrm{IU} / \mathrm{ml}$ \\
ESR & s & $0.0 \pm 13.3$ & $4-10 \times 109 / 1$ \\
CSF Protein & s & $0.36 \pm 0.31$ & Male: $0-10 \mathrm{~mm} / \mathrm{hr}$ \\
\hline
\end{tabular}

N: Number, SD: Standard Deviation, NHLS: National Health Laboratory Service

Table 3 Results of hypothesis $(\mathrm{H})$

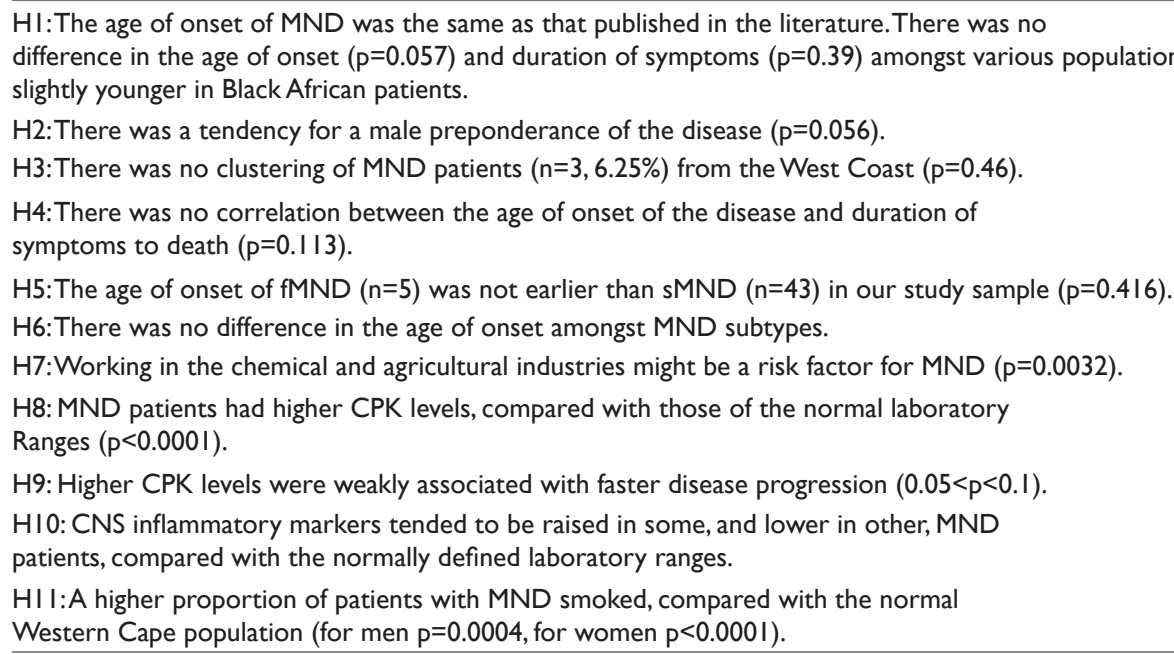

MND patient can present with bulbar, respiratory or spinal/limb onset. Bulbar-onset MND is said to have a worse prognosis compared to the other subtypes. ${ }^{6}$ In this study, there were no significant differences between all three subtypes of MND. The reason for the difference between our study and the other studies may relate to selection bias, i.e., the bulbar-onset disease may progress rapidly to death so that they might be poorly represented in our sample. Also, ascertaining the real time of onset of the illness is not easy, especially if the patient is anarthric. Furthermore, classification of the patients into one or the other group may not be straightforward especially if bulbar and limb symptoms started simultaneously.

Many studies have published an association between toxins and chemical exposure and the risk of MND. Most, however, have been inconclusive. The toxicity could be acute or slowly progressive over the years. An extensive review by Armon et al., ${ }^{7}$ concluded that exposure to lead and agricultural chemicals might be a risk factor for MND. ${ }^{7}$ It has been suggested that exposure to agricultural chemicals might explain the apparent association between MND. We compared the proportion of MND patients in our study, working in the agricultural chemical or chemical industry, with that known for the Western Cape population. Ideally, however, we should perform a case-control study in which age and geographically matched cases are compared to a similar population of controls (without MND). We could then compare the two groups of their occupations, both present, and past. Also, if these factors are important in MND, the exposure may be short-lived but very intense, and a short exposure, e.g., to heavy metals, chemicals or toxins, may be less well remembered than an extended period in a safer job. Conversely, there may be a recall bias. Patients with MND are more likely to remember their exposure to chemicals or toxins than those who are healthy and do not have MND. Our results, nevertheless, support the idea that chemical exposure might be an aetiological or risk factor for MND. It should, however, also be pointed out that potential patients from the principal agricultural districts of the Western Cape (Paarl, the Winelands, Franschhoek, lgin River Valley) are more likely to "drain" to the TBH. The association between agricultural chemicals and even possible cyanobacteria exposure in groundwater or wells may not be apparent in the population served by GSH.

CPK levels have been reported to be higher in MND than in the normal population. This finding was confirmed in our study. Chronic muscle denervation and atrophy would explain the rise in CPK: as muscles fibers disintegrate secondary to denervation, they release CPK, which enters the systemic circulation. Higher CPK levels are described in MND patients in the literature, but it was not clear that high CPK levels are associated with a faster progression of the disease to death. In this study, there was no association between CPK levels and disease progression. However, CPK levels could vary with disease duration. We might expect higher CPK levels with more progressive muscle wasting. More advanced cases, by definition, are nearer to death. Our results suggest that CPK levels are not reliable markers of disease progression and cannot be used as a prognostic factor.

These non-specific markers did not add much useful diagnostic information. They were both higher, and lower, in MND patients compared with the defined laboratory ranges. It might indicate that 
there was a systemic inflammatory component to the disease in some patients, but not in others. The numbers were too small to do posthoc analyses of a possible association between the degree of systemic inflammation and the progression of the disease. One could speculate that a more protective systemic inflammatory response might better "contain" the disease. On the other hand, a greater systemic inflammatory response could indicate more severe disease, with more significant "spill-over" of the pathology into the systemic circulation (Supplementary $1 \& 2$ ).

Armon concluded that smoking might be a risk factor for MND. ${ }^{7}$ Alonso evaluated the association between tobacco use and MND incidence and survival in a cohort study. ${ }^{8} \mathrm{He}$ found that the risk of MND was greater in smokers than non-smokers and that it was associated with poorer survival in women and not in men. We showed that the proportion of smokers in the MND cases was much higher than the rates of smoking in the Western Cape population in general. Our results support the hypothesis that cigarette smoking might be a risk factor for MND. Our data on whether a patient smoked or not was obtained from the clinical notes. We did not have accurate information about when they smoked, for how long they smoked, the number of pack-years or for how long they might have stopped smoking.

As this research is a retrospective study, not surprisingly, there was some missing information, and the clinician's notes often had to be interpreted. Naturally, if a prospective structured questionnaire were used for future new cases of MND, many of these problems could be circumvented. Some cases of MND might have been missed. A perfect but practically impossible study would have been a long-term cohort study with thousands of participants, who are normal at baseline, and who could be followed up prospectively over several years for the development of MND. In this way, one could ascertain the presence of actual risk factors, i.e., factors present in the participants that preceded the advent of the disease. Such a study would, however, be enormously expensive and labor-intensive. Since MND is a relatively rare condition, we would have needed to follow up a million participants to obtain 10 cases of MND. A more practical suggestion would be to combine the MND data of patients admitted to both GSH and those of the other main tertiary neurology unit in the Western Cape, i.e., at the TBH. If the same study were conducted over the same period in both hospitals, we would probably capture the majority of cases of MND in the Western Cape. We would then better be able to ascertain incidence and prevalence rates in the Western Cape. With larger numbers, many of our data that tended to significance, e.g., the younger age of onset of MND in African patients might become significant. Furthermore, we would be able to determine whether clustering occurs in the West Coast area. Despite these limitations, however, the study has been one of only a few conducted in our continent.

\section{Conclusion}

This study has reproduced many of the findings in the literature. The age of onset of the disease and male preponderance was similar.
Smoking and toxic chemical exposures were identified as possible risk factors. The disease may have an early age of onset in black Africans. This pilot study will serve as the basis for future larger prospective studies on MND prevalence and etiology in South Africa.

\section{Conflicts of interest}

Authors declare that there are no conflict of interests including employment, consultancies, stock ownership, honoraria, paid expert testimony, patent applications/registrations, and grants or other funding regarding my research.

\section{Acknowledgments}

None.

\section{Funding}

None.

\section{References}

1. Gea-Banacloche J, Johnson RT, Bagic A, et al. West Nile virus: pathogenesis and therapeutic options. Ann Intern Med. 2004;140(7):545-553.

2. Chopra M, Steyn N, Lambert V. Decreasing the Burden of Cardiovascular Disease. Western Cape Burden of Disease Reduction Project. 2007;6(7):51.

3. Imam I, Ogunniyi A. What is Happening to Motor Neuron Disease in Nigeria? Annals of African Medicine. 2004;3(1):1-3.

4. Zaldivar T, Gutierrez J, Lara G, et al. Reduced frequency of ALS in an ethnically mixed population. A population-based mortality study. Neurology. 2009;72(19):1640-1645.

5. Grunseich C, Rinaldi, C Fischbeck KH. Spinal and bulbar muscular atrophy: pathogenesis and clinical management. Oral Dis. 2014;20(1):6-9.

6. Li TM, Alberman E, Swash M. Comparison of sporadic and familial disease amongst 580 cases of motor neuron disease. J Neurol, Neurosurg Psychiatry. 1988;51(6):778-784.

7. Wijesekera LC, Leigh PN. Amyotrophic lateral sclerosis. Orphanet $J$ Rare Dis. 2009;4(1):3.

8. Armon C. An Evidence-Based Medicine Approach to the Evaluation of the Role of Exogenous Risk Factors in Sporadic Amyotrophic Lateral Sclerosis. Neuroepidemiology. 2003;22(4):217-228.

9. Alonso A, Logroscino G, Susan S, et al. Association of smoking with amyotrophic lateral sclerosis risk and survival in men and women: a prospective study. BMC Neurology. 2010;10(1):6. 